\title{
The chicken NKX2.8 homeobox gene: A novel member of the NK-2 gene family
}

Received: 28 January 1997 / Accepted: 8 February 1997

\begin{abstract}
We have isolated the chicken homeobox gene NKX2.8, which represents a novel member of the NK-2 gene family. Besides the homeodomain, the NKX2.8 protein contains two other conserved sequences, a TN and an NK2 domain. NKX2.8 is expressed in the ventral foregut, the developing heart, in the epithelial layers of the branchial arches and in the dorsal mesocardium. Thus, its expression overlaps partially, but also differs significantly from another chicken tinman orthologue, the NKX2.5 gene. It is suggestive that NKX2.8 and NKX2.5 play a cooperative role in early heart development.
\end{abstract}

Key words NK-2 homeobox $\cdot$ tinman $\cdot$ Heart · Branchial arch $\cdot$ Chicken

\section{Introduction}

The heart is the first functional organ in developing vertebrate embryos. It is the derivative of the earliest embryonic mesoderm generated during gastrulation, preceded only by extraembryonic mesoderm and endoderm (for review, see Lyons 1996). When the primitive streak has reached its full extension, the cardiac mesoderm forms two groups of cells on both sides of the rostral primitive streak which will fuse anterior of the axial mesoderm. With subsequent formation of the headfold and foregut pocket, the cardiac mesoderm becomes translocated to a ventral, more posterior position in association with the anterior intestinal portal. In the 1-3 somite stage embryo the determination of the two cardiac primordia takes place. As part of the splanchic mesoderm they develop in close contact with the endoderm of the forming foregut pocket at the anterior intestinal portal. They fuse from anterior to posterior thereby forming the single heart tube which undergoes further complex morphogenesis.

Edited by R. Balling

T. Boettger $\cdot$ S. Stein $\cdot$ M. Kessel (

Max-Planck-Institut für biophysikalische Chemie,

Am Fassberg, D-37077 Göttingen, Germany
This involves looping out to the right side and differentiation along the anteroposterior axis, with formation of the truncus arteriosus, ventricle, atrium and sinus venosus. In the vicinity of the heart, the pharynx develops pouches which alternate with the aortic arches connecting dorsal and ventral aorta. These blood vessels run within the branchial arches, which are prominent structures of the ventral cervical region of the embryo filled with mesenchymal cells derived from the neural crest.

Homeodomain proteins play an important role in regional specification, fate determination and organogenesis throughout embryogenesis. For the development of the dorsal vessel (the Drosophila heart), the homeobox gene tinman exerts a crucial function which is demonstrated by a complete lack of the heart in tinman null mutants (Bodmer 1993). The gene Nkx2.5 is a vertebrate orthologue of tinman and is expressed in developing hearts of fish, frog, chicken and mouse, thus representing a candidate for playing a key role in the specification of cardiac cells. However, mutation of the murine $\mathrm{Nkx} 2.5$ gene by targeting did not prevent the initial steps of heart development up to the formation of a straight heart tube, but impeded the looping of the heart tube and subsequent developmental steps (Lyons et al. 1995). Other tinman orthologues have been isolated from various vertebrate species and they are also transcribed during heart development. These include the $\mathrm{Nkx} 2.3$ genes isolated from fish, frog, chick and mouse (Harvey 1996), and the nkx2.7 gene so far only identified in zebrafish (Lee et al. 1996). All are candidates for participants in a combinatorial homeobox code specifying heart development.

Here we describe a further Nkx gene from chicken, NKX2.8, that shows a unique expression pattern in early heart development and in the branchial arches. We discuss its role in the specification of a ventral embryonic territory in combination with other homeobox genes.

\section{Materials and methods}

The NKX2.8 cDNA clone was isolated from a Hamburger-Hamilton stage 10 library (Charlebois et al. 1990) by low stringency 
A)

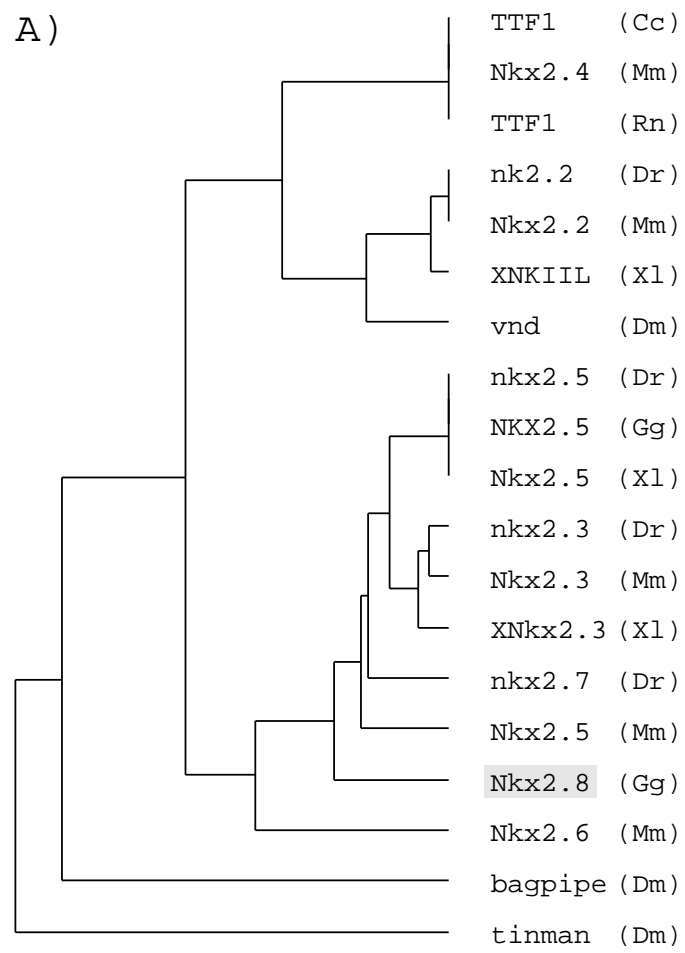

B)

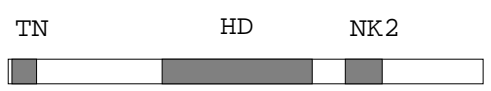

C) $\mathrm{TN}$ domains

\begin{tabular}{|c|c|}
\hline NKX2. 8 & TPFSVEDILSL \\
\hline NKX2. 5 & $\ldots \ldots k \ldots N$ \\
\hline $\mathrm{Nk} \times 2.5$ & $\ldots . . . K \ldots N$. \\
\hline$n k \times 2.5$ & $\ldots$...... \\
\hline NKX2. 5 & $\ldots . . . K \ldots$. \\
\hline $\mathrm{NK} \times 2.3$ & $\ldots K \ldots N$ \\
\hline$n k \times 2.3$ & $\ldots \ldots K \ldots K$. \\
\hline $\mathrm{nk} \times 2.7$ & ...K...K. \\
\hline TTF1 & $\ldots$...P \\
\hline bap & $\ldots$.....TR \\
\hline $\operatorname{tin}$ & $\ldots \ldots K \ldots N$ \\
\hline vnd & SG.HIS...N. \\
\hline
\end{tabular}

D) NK2 domains

E) Homeodomains

\begin{tabular}{|c|c|c|c|}
\hline NKX2. 8 & PRKVAVPVLVRNGKPC & $\mathrm{FE}$ & $(\mathrm{Gg}$ \\
\hline $\mathrm{NK} \times 2.5$ & $\ldots R I \ldots \ldots D \ldots$ & LG & \\
\hline $\mathrm{NK} \times 2.5$ & $\ldots R I \ldots \ldots D \ldots$ & LG & \\
\hline $\mathrm{nk} \times 2.5$ & . RIS. & LG & \\
\hline $\mathrm{NKX} 2.5$ & A.RI. . & LG & \\
\hline$x 2.3$ & . R. . & IG & \\
\hline & $\ldots \mathrm{D}$. & LT & \\
\hline & . R....... & HG & \\
\hline & . R.....KD. & $\mathrm{QA}$ & \\
\hline & R...... & LG & \\
\hline
\end{tabular}

Her

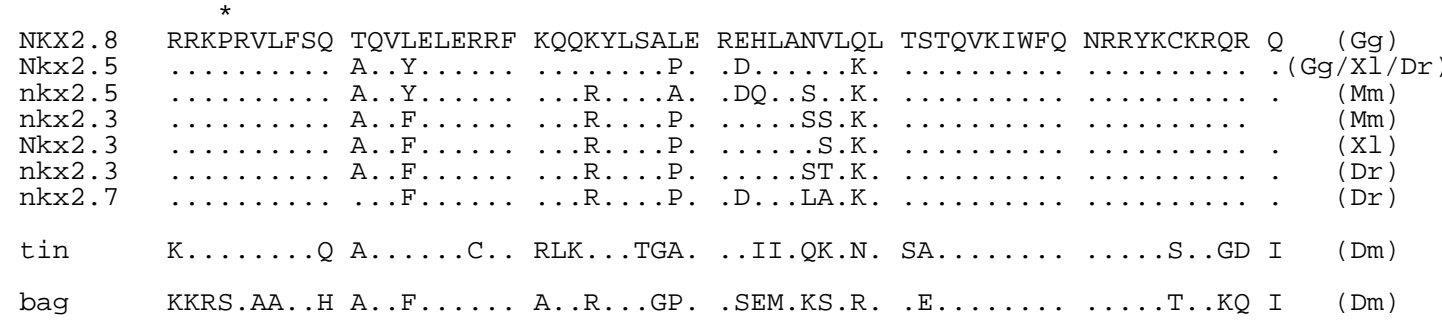

screening with a CNOT1 homeobox probe (Stein and Kessel 1995). The sequence of the 1111-bp cDNA clone has been submitted to the EMBL Database (Accession Number: Y10655).

For whole mount in situ hybridisation (Wilkinson 1992), we used a riboprobe of the entire NKX2.8 cDNA. We also used riboprobes of either the $3^{\prime}$ untranslated region or the open reading frame with its conserved sequences. All probes gave the same spatio-temporal expression pattern.

\section{Results and discussion}

\section{The NKX2.8 protein}

We have isolated a cDNA clone with an open reading frame for a homeodomain protein of 193 amino acids. The clone encompasses 31 bp of $5^{\prime}$ untranslated sequence upstream of a putative start codon and a 467-bp $3^{\prime}$ trailer including a polyadenylation signal and a polyA tail.

The homeodomain sequence shows that the gene belongs to the NK-2 family of homeobox genes, of which members from Drosophila and vertebrates have been isolated. It shows $91.8 \%$ identity to the homeodomains of the chicken, Xenopus and zebrafish Nkx2.5 genes,

Fig. 1A-E Comparative analysis of NK-2 protein domains. A Pileup of the NK-2 homeodomains (Wisconsin Package of Genetics Computer Group). Note that the vertebrate central nervous system (CNS) genes (TTF-1 etc.) and the Drosophila vnd gene form a group of closely related genes. Also the vertebrate heart genes (NKX2.5 etc.) form a separate group. However, the Drosophila genes involved in heart and gut formation are more distant and do not appear together with the vertebrate genes. B Schematic structure of the NKX2.8 protein. C Comparison of the TN domains. Note the unique positions in NKX2.8. D Comparison of the NK2 domains. Note the unique positions in NKX2.8. E Comparison of the NK2 homeodomains. Note the proline in position $4(*)$ and unique positions in NKX2.8 (Dm Drosophila melanogaster, Dr Danio rerio, Gg Gallus gallus, Mm Mus musculus, Rn Rattus norvegicus, Xl Xenopus laevis)

90\% identity to the Xenopus Nkx2.3 gene and $88.5 \%$ identity to the zebrafish nkx2.7 gene. It is equally distant from other NK-2 gene types, hence we named the new isolate "NKX2.8" (Fig. 1A). Its homeodomain contains a tyrosine at position 54 which is characteristic for all members of the NK-2 family (Fig. 1E). Another striking feature is a proline at position 4 . This is encoded in a number of homeobox genes outside the NK-2 family, but within that family it is shared only between 
the subgroup of genes expressed in the heart and in the cnidarian gene CnNK-2 (Grens et al. 1996). It was shown that the flanking, highly conserved amino acids at position 3 and 5 are important for the contact of the homeodomain to its target DNA (Kissinger et al. 1990). One can assume that the presence of a proline between these two residues should alter their contact specificity significantly.

As well as the homeodomain, the predicted NKX2.8 protein contains two domains typically conserved in NK2 class homeobox genes: the TN domain near the $\mathrm{N}$ terminus and the NK2 domain located at the $\mathrm{C}$ terminus of the homeodomain (Fig. 1B; Harvey 1996; Smith and Jaynes 1996). We did not find a C-terminal GIRAW-motif, which is present in the $\mathrm{Nkx} 2.3$ and $\mathrm{Nkx} 2.5$ genes, and also in zebrafish nkx2.7 in a less conserved form. Sequence comparison of the TN and NK2 domains strongly supports the notion that NKX2.8 cannot be grouped into one of the known orthologue classes (Fig. 1C, D). Thus, we conclude that we have cloned the cDNA of an up to now unknown type of NK-2 gene.

\section{Expression of NKX2.8}

The first expression of NKX2.8 is symmetrically detectable in the lateral endoderm of the anterior intestinal portal in the newly formed headfold at Hamburger-Hamilton stage 6 (Fig. 2A; HH st.6; Hamburger and Hamilton 1951). In the two-somite stage embryo (HH st.7) the lateral endodermal expression domains expand anteriorly and fuse around the anterior intestinal portal (Figs. 2B, $3 \mathrm{~A}-\mathrm{C}$ ). NKX2.8 transcripts are now detected in the ventral foregut pocket, i.e. the prospective pharyngeal endoderm. At HH st.8 (4 somites), NKX2.8 is also mesodermally expressed in the myocardium of the developing heart tubes (Fig. 2C). The myocardial expression domain is retained as the forming heart tubes elongate and then merge (HH st.11). In this phase, the most intense staining is on the proximal vitelline veins, so that NKX2.8 predominantly characterises the posterior portion of the

Fig. 2A-E Whole mount in situ analysis of NKX2.8 gene expression. A HH st.6 (Hamburger-Hamilton stage 6; headfold stage). Note expression in the lateral endoderm of the headfold. B HH st.7 (2 somite stage). Transcripts are found in the endoderm around the anterior intestinal portal. Bars indicate the levels of sections shown in Fig. 3. The upper bar refers to Fig. 3A, the middle bar to Fig. 3B, the lower bar to Fig. 3C. C HH st.8 (5 somite stage). Transcripts are detected in the ventral foregut and the cardiac mesoderm. D HH st.12 (15 somite stage). Expression is visible in the myocardium of the heart tube, the vitelline veins and in the middle part of the ventral foregut. Bars indicate the levels of sections shown in Fig. 3 of a comparable embryo. The upper bar refers to Fig. 3D, the middle bar to Fig. 3E, the lower bar to Fig. 3F. E HH st.15 (24 somite stage). NKX2.8 is detected in the branchial arches and in the dorsal mesocardium. Bars indicate the levels of sections shown in Fig. 3. The left bar refers to Fig. 3G, the middle bar to Fig. 3H, the right bar to Fig. 3I. (aip anterior intestinal portal, $b a$ branchial arches, $\mathrm{cm}$ cardic mesoderm, $\mathrm{dm}$ dorsal mesocardium, $n$ node, $v v$ vitelline veins; bar in $A$ indicates $300 \mu \mathrm{m}$ in $\mathbf{A}$ and $\mathbf{B}, 210 \mu \mathrm{m}$ in $\mathbf{C}$ and $\mathbf{D}, 330 \mu \mathrm{m}$ in $\mathbf{E})$
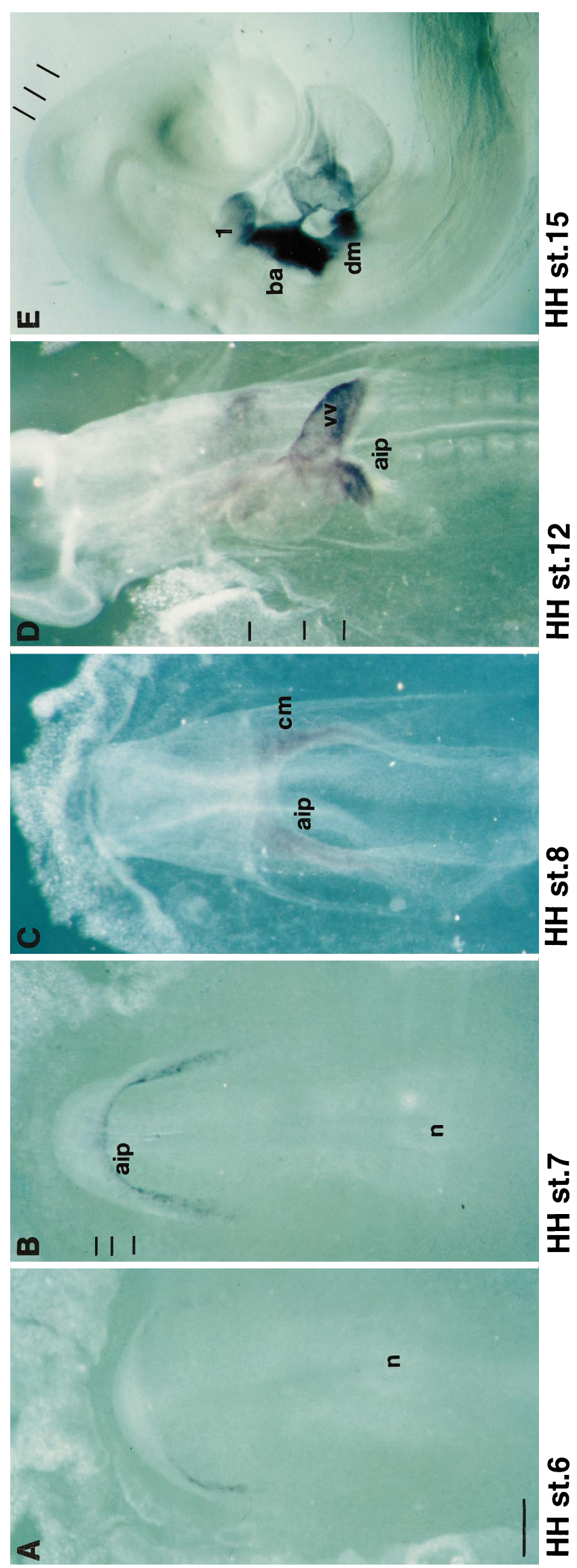
HH st.7

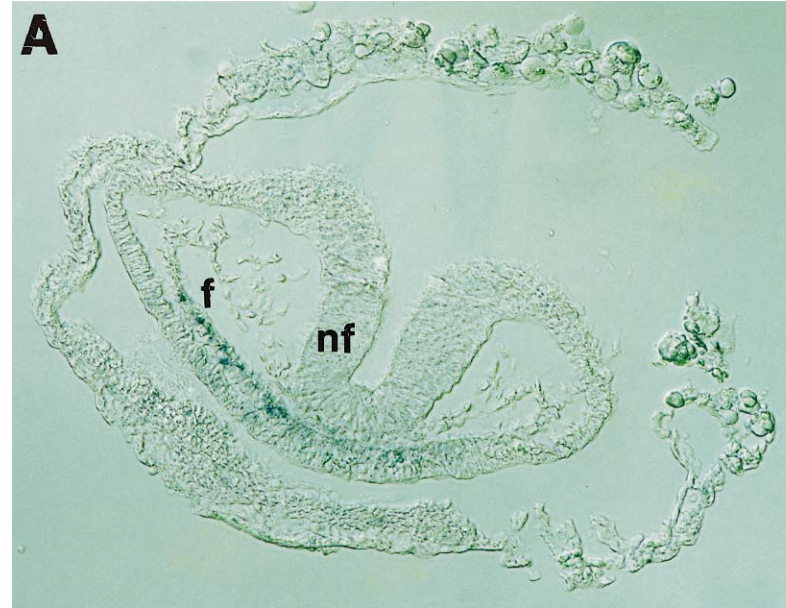

B
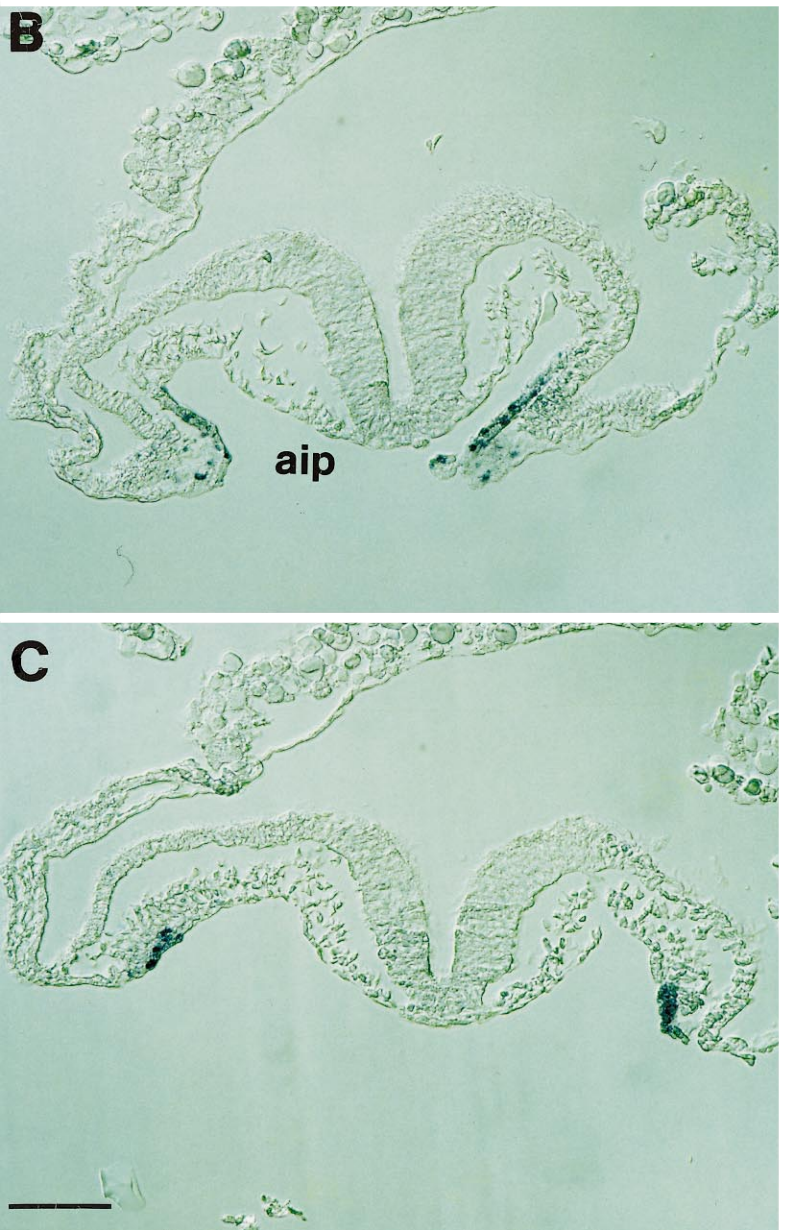

Fig. 3A-I Transverse sections of embryos after whole mount in situ analysis with NKX2.8. A-C HH st.7 embryo. A Note expression in the ventral endoderm of the foregut pocket. B Note expression only in the lateral endoderm of the anterior intestinal portal. C Note expression in the lateral endoderm before foregut formation. The levels of the cross sections are indicated in Fig. 2B. D-F HH st.11 embryo. D Note transcripts in the middle part of the ventro-lateral foregut pocket and the myocardium of the forming heart tube. E Note transcripts in the epimyocardium (em), but not the endocardium $(e c)$. F Note expression in the vitelline veins $(v v)$.
HH st.11

HH st.15
D

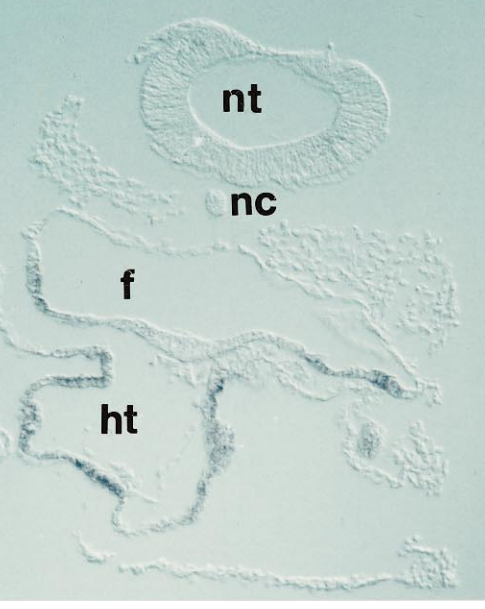

$\mathbf{E}$

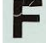

G

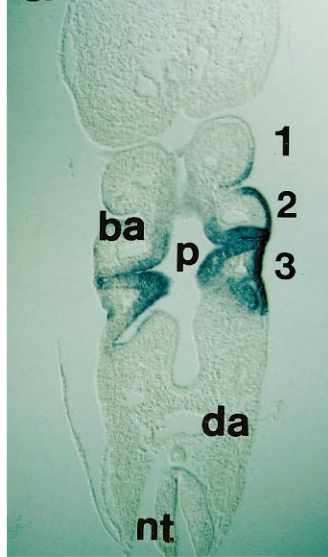

H
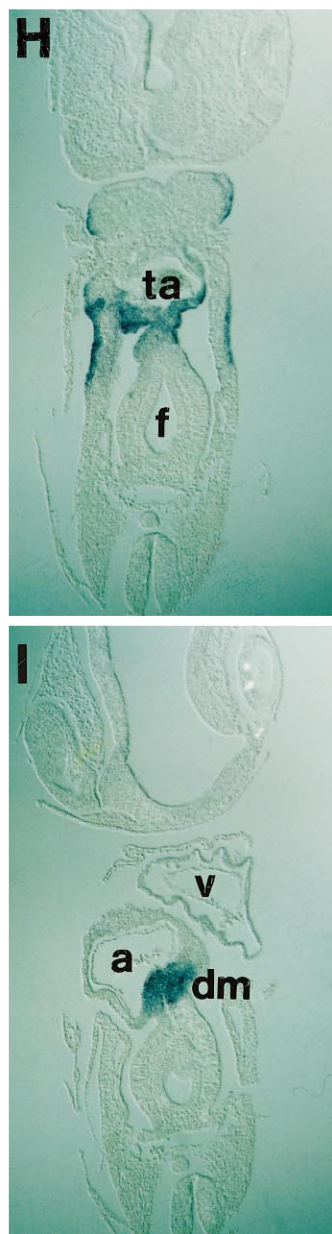

The levels of the cross sections are indicated in Fig. 2D. H-I HH st.15 embryo. G Note expression in the epithelia of the branchial arches. H Note expression in the distal part of the truncus arteriosus. I Note expression in the dorsal mesocardium. The levels of the horizontal sections are indicated in Fig. 2E ( $a$ atrium, aip anterior intestinal portal, $b a$ branchial arches (1-3), $d a$ dorsal aorta, $d m$ dorsal mesocardium, ec endocardium, em epimyocardium, $f$ foregut, $h t$ heart tube, $n c$ notochord, $n f$ neural folds, $n t$ neural tube, $p$ pharynx, $v$ ventricle, $v v$ vitelline veins; bar indicates $70 \mu \mathrm{m}$ in A-F, $202 \mu \mathrm{m}$ in $\mathbf{G}-\mathbf{I}$ ) 


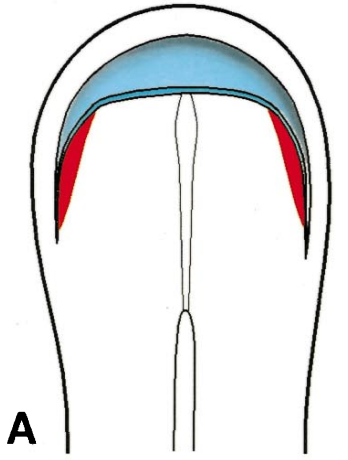

Fig. 4A-C Comparison of NKX2.8 and NKX2.5 expression in chicken. A At the headfold stage (HH st.6) NKX2.8 (red) and NKX2.5 (blue) transcripts are located in different domains around the anterior intestinal portal (aip); whereas NKX2.8 is located in the lateral endoderm of the aip, NKX2.5 is expressed in the lateral mesodermal plates, in the anterior endoderm and ectoderm. B At the 12-somite embryo (HH st.11), both genes are expressed in the myocardium and the ventral foregut. NKX2.8 expression is especially strong in the vitelline veins and exhibits a restricted domain in the foregut. C In 24-somite embryos (HH st.15) the expression domains of both genes are completely divergent: NKX2.8 is expressed in the epithelia of the branchial arches and in the dorsa mesocardium, whilst NKX2.5 is expressed solely in the heart. The yellow circle marks the ventral territory consisting of the ventral forebrain, ventral foregut, heart and branchial arches

heart. Endodermal expression at $\mathrm{HH}$ st. 11 is restricted to the middle part of the developing foregut in ventro-lateral domains (Figs. 2D, 3E-G). As the heart tube bends, NKX2.8 expression starts to fade from the myocardium. By HH st.15, there is no expression left in the myocardium of the heart. The endodermal expression increases strongly in the second and third pharyngeal pouch. High levels of NKX2.8 transcripts are also present in the ectodermal epithelium of the second and third branchial arch, whereas lower levels occur distally in the first arch. The aortic arches and the branchial arch mesenchyme remain negative. A strong expression domain is visible in the dorsal mesocardium and there is expression in the anterior part of the truncus arteriosus.

The described expression pattern differs significantly from the patterns of all other NK-2 genes, further supporting our conclusion that NKX2.8 is a new NK-2 gene type. Most noteworthy are differences between NKX2.8 and NKX2.5 expression (Fig. 4; Schultheiss et al. 1995) NKX2.5 is expressed earlier than NKX2.8, demarcating initially the lateral cardiac mesoderm, but not the lateral endoderm (HH st.6). NKX2.5 transcripts are found along the whole rostrocaudal extent of the heart tube and of the ventral pharynx. NKX2.5 is not expressed in the pharyngeal pouches and branchial arches and at later stages it shows expression solely in the heart. It is intriguing that the Nkx2.5 mutant mice phenotype appears at a stage comparable to the point when NKX2.8 expression disappears from the heart, whereas in wildtype Nkx2.5 remains strongly expressed in the heart tube (Lyons et al. 1995).

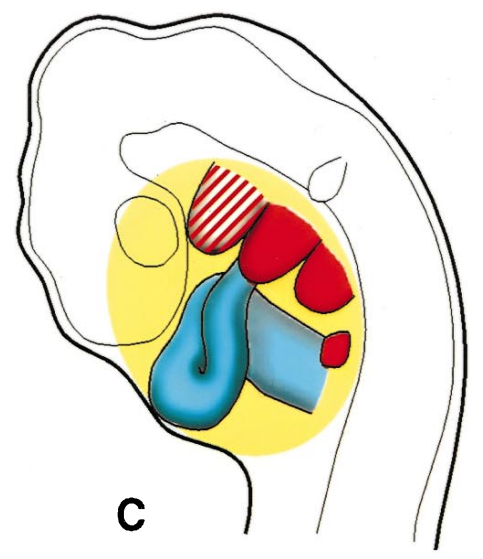

A ventral NK-2 territory?

Recently it has been shown that an NK-2 gene is involved in specifying the basal endoderm in hydra (Grens et al. 1996). An endodermal expression pattern is a hallmark of nearly all members of the NK-2 gene family. However, many NK-2 genes have another predominant expression domain in a different germ layer, justifying a further subdivision of the NK-2 family into two major branches. One group of genes is expressed in the central nervous system (CNS), including the Drosophila vnd and the vertebrate Nkx2.1 and Nkx2.2 genes (Harvey 1996). This suggests that one ancestral NK-2 gene already had a function in the development of the nervous system before the divergence of deuterostomia. The other group of vertebrate NK-2 genes is expressed in the developing heart. However, they are clearly distant from both the Drosophila heart gene tinman and from bagpipe. Vessels in the gut musculature have been proposed to be the origin of the heart of vertebrates as well as of invertebrates (Harvey 1996). We propose that tinman and the respective vertebrate genes evolved independently from an NK2 gene expressed in the gut musculature. This ancestral gene was possibly characterised by the proline in position 4, typical of the homeodomains in tinman and vertebrate heart NK-2 genes. A further indication to the independent development of vertebrate heart $\mathrm{Nkx} 2$ genes and tinman could be the observation that tinman lacks an NK-2 domain. Functional subdivisions in the vertebrate heart occurred during the process of evolution, for which the duplication of the $\mathrm{Nkx} 2$ genes leading to $\mathrm{Nkx} 2.3$, $2.5,2.7$, or 2.8 , may have been a prerequisite.

It is interesting that the expression domains of the vertebrate NK-2 genes together form a coherent territory consisting of ventral forebrain, ventral foregut, the ventrally protruding branchial arches and the heart (Fig. 4C). As an analogy to the trunk as a posterior Hox gene territory (Kessel and Gruss 1991), a ventral Nkx territory may represent a unit with unique morphoregulatory properties.

Acknowledgements We thank K. Ewan, L. Lemaire, E. Pera and T. Roeser for discussions. The cDNA library was kindly provided by R. Grainger. The work was supported by the Max-Planck-Gesellschaft and the DFG by the Graduiertenkolleg "Molekulare Genetik der Entwicklung". 


\section{References}

Bodmer R (1993) The gene tinman is required for specification of the heart and visceral muscles in Drosophila. Development 118: 719-729

Charlebois TS, Henry JJ, Grainger RM (1990) Differential cytokeratin gene expression reveals early dorsal-ventral regionalization in chick mesoderm. Development 110: 417-425

Grens A, Gee L, Fisher DA, Bode HR (1996) $C n N K-2$, an $N K-2$ homeobox gene, has a role in patterning the basal end of the axis in Hydra. Dev Biol 180: 473-488

Hamburger V, Hamilton HL (1951) A series of normal stages in the development of the chick embryo. J Morphol 88: 49-92

Harvey RP (1996) NK-2 homeobox genes and heart development. Dev Biol 178: 203-216

Kessel M, Gruss P (1991) Homeotic transformations of murine vertebrae and concomitant alteration of Hox codes induced by retinoic acid. Cell 67: 89-104

Kissinger CR, Liu BS, Martin-Blanco E, Kornberg TB, Pabo CO (1990) Crystal structure of an engrailed homeodomain-DNA complex at 2.8 A resolution: a framework for understanding homeodomain-DNA interactions. Cell 63: 579-590
Lee K-H, Xu Q, Breitbart RE (1996) A new tinman-related gene, nkx2.7, anticipates the expression of $n k x 2.5$ and nkx2.3 in zebrafish heart and pharyngeal endoderm. Dev Biol 180: 722731

Lyons GE (1996) Vertebrate heart development. Curr Opin Gen Dev 6: 454-460

Lyons I, Parsons LM, Hartley L, Li R, Andrews JE, Robb L, Harvey RP (1995) Myogenic and morphogenetic defects in the heart tubes of murine embryos lacking the homeo box gene Nkx2-5. Gen Dev 9: 1654-1666

Schultheiss TM, Xydas S, Lassar AB (1995) Induction of avian cardiac myogenesis by anterior endoderm. Development 121: 4203-4214

Smith ST, Jaynes JB (1996) A conserved region of engrailed, shared among all en-, gsc-, NK1-, NK2- and msh-class homeoproteins, mediates active transcriptional repression in vivo. Development 122: 3141-3150

Stein S, Kessel M (1995) A homeobox gene involved in node, notochord and neural plate formation of chick embryos. Mech Dev 49: 37-48

Wilkinson DG (1992) In situ hybridisation: A practical approach. Oxford University Press, Oxford London 